\title{
HOW CAN HYDROGEN BECOME A M METAL?
}

HIGH-ENERGY-DENSITY PHYSICS DESCRIBES SOME OF THE MOST EXTREME ENVIRONMENTS IN OUR UNIVERSE, LIKE THE CORES OF STARS. DR VALENTIN KARASIEV AND DR SUXING HU OF THE LABORATORY FOR LASER ENERGETICS AT THE UNIVERSITY OF ROCHESTER IN THE US ARE CREATING NEW MODELS TO DESCRIBE THESE EXOTIC PROCESSES

\section{TALK LIKE A PHYSICIST}

\section{THEORETICAL/COMPUTATIONAL}

MODEL - a series of equations that describe and can predict the behaviour of a system. These are often very complex so are carried out on a computer rather than doing them by hand

\section{HIGH-PERFORMANCE COMPUTING - using large, very} powerful computers to run simulations

NUCLEAR FUSION - when two or more atoms are joined together to form a new element

ELECTRONS - negatively charged particles that are part of atoms

PLASMA - another state of matter like solids, liquids and gases. Plasmas are usually very hot clouds with electrons that may behave very differently to other phases of matter
At very high temperatures and pressures, physics as we know it starts to change. The study of such environments is known as high-energy-density physics. These extreme environments are found at the hearts of our sun, and in the interior of giant planets and various types of stars. While they might seem far away, these environments could hold the secret for understanding processes like nuclear fusion which could be an incredibly important source of energy in the future if we could find a way to control the process on Earth.

Dr Valentin Karasiev and his colleague, Dr Suxing $\mathrm{Hu}$, of the Laboratory for Laser Energetics at the University of Rochester are experts in developing theoretical and computational models to describe highenergy-density processes. One of the big challenges they face in their research is that many models which describe the physics of materials at zero to near-room temperatures stop working under such extreme conditions. To overcome this, they need not only to create new models, but to implement the models in a computer code and find ways to run them efficiently using high-performance computing.

While Valentin only works using theoretical approaches, he emphasises how important both experiments and calculations are in this area of physics. "Comparisons with experiments can validate theoretical methods used for predictions." However, having very good theoretical predictions can do more than just match experiments. Valentin says, "Theoretical predictions, which are easier and faster to produce compared to experimental measurements, can be used to guide experiments."

But how can we study the physics of extreme environments on Earth? Valentin says, "To create high pressures, scientists can perform diamond anvil cell experiments, when a small sample is compressed between two diamond tips or perform gas-gun experiments when a high-velocity projectile impacts a target to create high pressure. For high pressures and temperatures, powerful laser beams are used to heat and compress a target."

\section{STRANGE METALS}

One of the projects Valentin and Suxing have been working on involves looking at hydrogen. Hydrogen, as we know it on Earth, is a colourless, light gas. It is also the gas that acts as a fuel for nuclear fusion in stars that keeps 


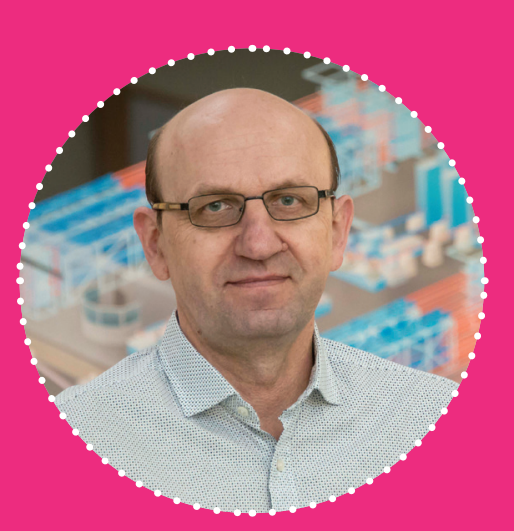

DR VALENTIN KARASIEV

Scientist

Laboratory for Laser Energetics

University of Rochester, USA

$\ldots \ldots \ldots \ldots$

\section{FIELD OF RESEARCH}

High-Energy-Density Physics

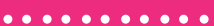

\section{RESEARCH PROJECT}

Developing methods for modelling processes and physics that occur in extreme environments.

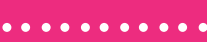

\section{FUNDERS}

This work was supported by U.S. National Science Foundation PHY Grant No. 1802964, the

Department of Energy National Nuclear Security Administration under Award No DE-NA0003856,

the University of Rochester, the New York State Energy Research and Development Authority, and the National Energy Research Scientific Computing

Center, a DOE Office of Science User Facility supported by the Office of Science of the U.S. Department of Energy under Contract No. DEAC02-05CH11231.

We usually think of metals as hard, shiny materials but what really defines a metal is how the electrons in the material behave. Insulating materials, like wood and gaseous hydrogen, do not conduct electricity. This is because the electrons in the material are bound and do not start to move and flow when the material becomes part of a circuit.

In metallic hydrogen, the electrons are shared between different hydrogen sites and can flow freely through the network of atoms. This means the hydrogen has become a conductor, and scientists hint that this may have some exciting implications where, "under high pressure hydrogen and/or some hydrogen rich materials may become room temperature superconductors."

A superconductor is a material that lets electrons move with no resistance. For most normal conducting metals, an electron experiences some resistance to its movement and so generates heat as it moves. This means energy is constantly being wasted and is part

of why some electronic devices get hot when in use.

In a superconductor, this heating does not happen and so no energy is wasted. If a perfect superconducting device was made, the device could be on nearly forever, without ever depleting the battery. Recently, Suxing's and Valentin's colleagues, the experimental group of Professor Ranga Dias, discovered room temperature superconductivity in carbonaceous sulphur hydride (CSH) systems and from Suxing's calculations they were able to identify which structures would be the most stable room-temperature superconductors under high pressures.

\section{DEVELOPING METHODS}

One of the biggest challenges in doing these types of calculations for Valentin is that he often needs to develop the methods so they can be applied to model extreme environments. Many of the practical methods using quantum mechanics have not been developed to account for the high temperatures of these extreme environments.

Valentin has made several key developments in the advancement of density functional theory-based methods for high-energy- density environments to make them efficient enough and usable to describe systems such as warm dense hydrogen. As well as leading these developments he has applied them to problems such as warm dense silicon plasmas and finding a way to describe how unusual interactions between atoms can arise, with unusual behaviour of electrons, in extreme environments.

In the future, Valentin and Suxing will be looking to develop more reliable and faster ways to describe the behaviour of materials for nuclear fusion experiments and mixtures of elements important for astrophysics and chemistry under high pressures. They consider this an exciting time to be in high-energydensity physics as it becomes increasingly involved with many other areas of science. As computers become faster, this will also make it possible to describe even more complex and exciting systems and to really understand the wealth of physics going on in the hearts of our stars. 
C

\section{ABOUT HIGH-ENERGY-DENSITY PHYSICS}

High-energy-density physics is an exciting and rapidly developing field all about trying to understand the physics of what happens at extreme conditions and very high energy densities. Many people working in this area are researchers across different sub-disciplines. In high-energy-density physics, some people specialise in laser experiments to use very powerful laser beams to generate extreme conditions in materials. Other people specialise in plasmas and understanding how they form and interact with lasers, and how the electrons behave.

All of these are very exotic and unusual types of physics that we do not encounter in our everyday lives. However, we do use plasmas to help make circuit boards for electronics and we rely on our stars performing nuclear fusion to act as a source of heat and light. Once, it was these nuclear fusion processes that formed the heavier elements that we find on Earth, such as iron.

Working in high-energy-density physics is a specialised area of physics. Generally, people like Valentin and Suxing start with a more general degree in physics and become more specialised over time. There is lots of international activity in the field, with new experiments being developed all the time to recreate these extreme conditions on Earth and explore all of the new physics to be discovered there.

\section{WHAT MOTIVATES YOU TO DO THE WORK YOU DO?}

"My motivation is a passion for science as it provides a basis for understanding and discovering unknown, unusual and often counterintuitive properties of matter," says Valentin. "I also like to develop theory and new methods, I like to write codes and run simulations and really enjoy my work. Getting to mentor graduate students and completing and publishing research projects are also very rewarding parts of the job."

WHAT TYPES OF COLLABORATION DOES YOUR WORK INVOLVE?

"We collaborate with theory groups at other research institutions. Modern science is complicated, and sometimes the efforts of several collaborators are required to make key advances. We also frequently collaborate with experimental groups as the measurements the experimentalists make allows for crossvalidation of theoretical predictions."

WHAT CHALLENGES DO YOU THINK THE NEXT GENERATION OF PHYSICISTS WILL BE FACING?

"In my opinion, the increasing multidisciplinary character of physics is one of the biggest challenges. This is especially true for materialsrelated research. For example, nowadays one may be an expert in materials science and have just some knowledge of computational science, perhaps just enough to run simulation packages and sometimes only with a general knowledge of theory/methods behind implementations in these packages. However, with the increasing importance of computer simulations and development of faster and more powerful supercomputers, even specialists in materialsrelated topics will need to become experts with deep knowledge in computer science and mathematics. While challenging, all of these developments may change the paradigm of how research is conducted drastically, increasing the leading role of theoretical modelling and predictive simulations."

\section{EXPLORE A CAREER IN HIGH-ENERGY- DENSITY PHYSICS}

- There are roles for both experimentalists and theoreticians in high-energy-density physics. In both cases, a strong general physics background is essential and good mathematical skills will also help.

- National laboratories and universities are where the majority of research in high-energy-density physics is and there are a large number of subtopics and disciplines that can be specialised in.

- The Laboratory for Laser Energetics - where Valentin and Suxing are based - runs a Summer Research Program for High School Juniors. Find out more:

www.lle.rochester.edu/index.php/education/ summer-research-program-for-high-school-juniors/
- Salaries range with experience, research institution and country, but start around $\$ 40,000$ and, with experience, can be in excess of $\$ 80,000$.

\section{PATHWAY FROM SCHOOL TO HIGH-ENERGY-DENSITY PHYSICIST}

Valentin recommends studying chemistry, physics and calculus/mathematics at school. At university, study physics or maths and take classes in quantum chemistry and quantum mechanics.

For theoretical physics job and many experimental ones, knowledge of computer programming languages and coding experience will also be beneficial. Much of Valentin's research involves implementing new theoretical developments in new or existing codes and optimising them for high-performance computing set-ups.

Nearly all physicists will have a bachelor's degree in physics or maths. In some areas of physics, a degree in engineering or chemistry may also be suitable. For a research position in a university, like Valentin and Suxing, a relevant master's and $\mathrm{PhD}$ is also important. If you want to specialise in high-energy-density physics, this will usually be done at master's or PhD level. 


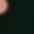

WHO OR WHAT INSPIRED YOU TO BECOME A SCIENTIST?

"My high school physics teacher, as well as my older brother, who is also physicist. I have had a strong passion for math and physics since high school and, from 5 th grade, I was interested in radio-electronics."

WHAT ATTRIBUTES HAVE MADE YOU SUCCESSFUL?

"The key attributes for being a successful scientist include being good at abstract thinking, as well as learning how to perform a sequence of logical deductions. This involves starting with assumptions and established work and seeing what can be deduced to lead to conclusions about a problem. It takes a kind of courage and imagination to put forward new or non-standard hypotheses."

WHAT ARE YOUR PROUDEST CAREER ACHIEVEMENTS?

"One of my proudest career moments was my move to my current laboratory,

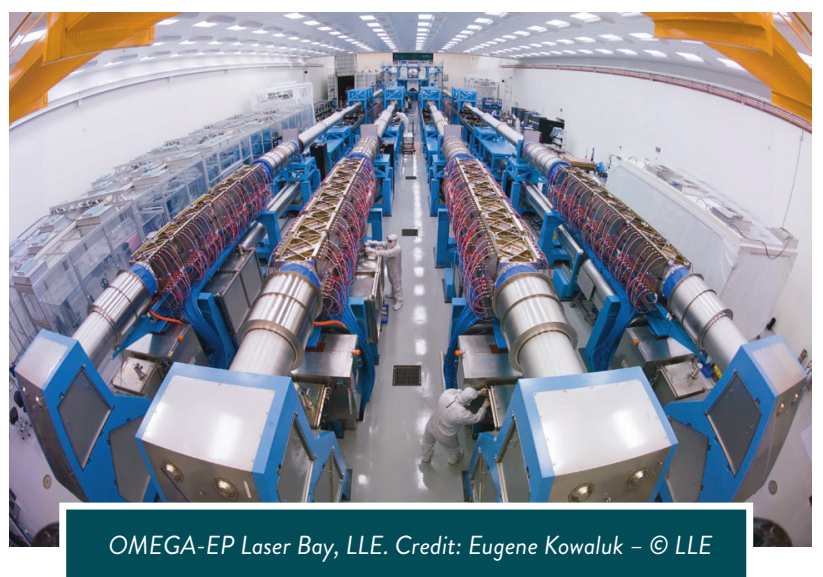

the Laboratory for Laser Energetics at the University of Rochester, three and a half years ago. I have moved several times in my career, from Russia to Venezuela to the USA, and each step has presented new opportunities to learn and new challenges to overcome."

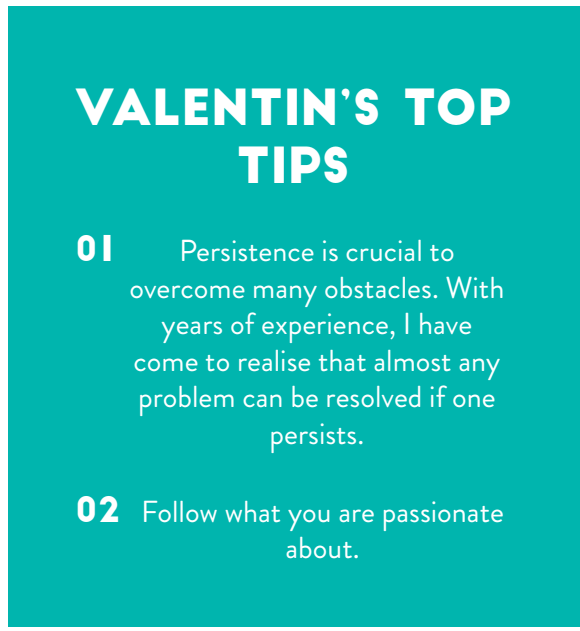

